\author{
Military Technical College \\ Kobry El-Kobba \\ Cairo, Egypt
}

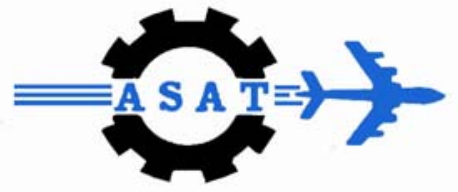

12-th International Conference

on

Aerospace Sciences \&

Aviation Technology

\title{
PHOTOELASTIC ANALYSIS OF STRESS TRANSFER FROM MATRIX TO THE FIBRE THROUGH AN INTERPHASE IN COMPRESSION
}

\author{
Jawad Ahmad Nisar*, Constantinos Soutis**, Frank R. Jones*** \\ Department of Mechanical Engineering, Sheffield University, Mappin Street, Sheffield, S1 3JD, UK
}

\begin{abstract}
The objective of the current work is to examine the stress transfer in a (a limiting design parameter in aircraft composite structures) Glass Fibre Reinforced Polymer (GFRP) composite taking into account the fibre/matrix interphase in compression, observe the stability of the interphase region during compressive loading and study the transfer of stress from matrix to the fibre through this region. In this study, a GFRP composite system is used. The composite material is made of a coated glass fibre (with a low modulus, "LM" and high modulus "HM" interphase) as a reinforcement surrounded by a polymer matrix. A finite element analysis for a single short fibre with two different Interphases (LM and HM) embedded in a block of polymer matrix is performed and interfacial shear stress results are compared to experimental data (photoelasticity) and existing analytical solutions.
\end{abstract}

Key Words: Glass Fibre, Interphase, Interfacial Strength, Debonding, Compressive Loading, 3-Dimentional Finite Element Analyses, Photoelasticity.

* Graduate Student, Department of Aerospace Engineering, Sheffield University.

** Professor, Department of Aerospace Engineering, Sheffield University.

$\star \star \star$ Professor, Department of Engineering Materials, Sheffield University 


\section{Introduction}

The fibre-reinforced composite consists of fibre tows containing individual fibre with typical diameters of $0.004-0.100 \mathrm{~mm}$ held together with a matrix which envelopes the fibre [5]. The performance of such fibre composites are excellent particularly with specific strength equating to four times that of steel but with density two thirds that of aluminum [4]. A layer of composite material is defined as a lamina and stacking lamina forms an FRP laminate. FRP has been used for many years in the aerospace and automotive industries and has recently been used in mechanical structures as an alternative to metal. Engineers are exploiting the advantages of using FRP; advantages such as light weight, corrosion resistance, low thermal and electrical conductivity, high strength to weight and stiffness to weight ratios, and the ability to vary the properties over a wide range of values. The strength and stiffness of fibrereinforced composites depend very much upon the properties of the interface/interphase region between the matrix and the fibre surface. The primary function of the fibre/matrix interface is to transmit stress from the polymer matrix to the reinforcing fibre. The ability to transmit stress across the phase boundary depends upon the mechanical properties of the matrix, the load-bearing ability of the fibre and the thickness and strength/stiffness of the fibre-matrix interface/Interphase. This research is motivated by previous studies done on the compressive behavior of interface/interphase and load (Stress) transfer through an interface/interphase in Fibre reinforced polymers (GFRP) laminated composite. The interface/interphase is the region where the fibre and matrix meet. This formation of region has a unique performance, composites can be attributed the interface. The fibre/matrix interface has a considerable influence on the structural veracity of a fibrous composite. For example the stronger the interface bond, the higher is the composites static strength. A strong interface bond also yields a brittle and weak interface results in a higher fracture toughness composites. When a composite is loaded compressively in the longitudinal direction failure can occur by a number of mechanisms including buckling, transverse splitting and interfacial shear. In addition to structural elastic buckling the fibre can undergo micro buckling that can lead to internal failure. Fiedler and Schulte [2] used photoelastic analysis to determine the interfacial shear stress and tensile stress in a glass fibre/epoxy resin in a fibre fragmentation. It was shown that the interfacial shear stress reached a maximum near to the fibre end. Anbu Clemension Johnson [4] works on Modeling of stress transfer through an interface/interphase in Fibre reinforced polymer composite. His research work is very informative. He proofs his work using FEA modeling and experimental verification.

In this study Glass Fibre reinforced polymers (GFRP) Composite is used. Composite material made of coated (LM and HM interphase) Glass fibre as a reinforcement surrounded by a solid matrix. Finite element model for a single short fibre with two interphase (LM and HM) modeled in the matrix and compare their interfacial shear stress in compression. Single short fibre test specimen is fabricated with (LM and HM) interphase embedded in a polymeric matrix and experimental testing is performed. In this experiment Phase stepping photoelasticity technique is used to calculate the interfacial shear 
stress in tension and compression. Finally the results from FEA interfacial shear stress (LM and HM) compared with photoelasticity interfacial shear stress value (LM and HM) in compression. In compressive loading due to fibre misalignment and buckling, the efficiency of stress transfer is affected from matrix to fibre before the interfacial failure. Therefore Fibre/Matrix interphase changes the composite compressive strength.

\section{Experimental Methods}

\subsection{Specimen Fabrication}

For the preparation of the short fibre composite specimen initially needs two steps. Firstly make silicon rubber female mould and secondly to make coated fibre with different types of interphase (LM and HM). Materials used for making these interphase are

\begin{tabular}{|l|l|}
\hline \multicolumn{1}{|c|}{ LM Interphase } & \multicolumn{1}{|c|}{ HM Interphase } \\
\hline Epoxy resin : Shell Epicote 828 & Epoxy resin : 828 \\
\hline Flexibilizer ( Aliphatic Epoxy resin) : Araldite GY 298 & \\
\hline Hardener : Nadic Methylene Anhydride (NMA) & Hardener BDMA/NMA \\
\hline
\end{tabular}

\subsection{Silicon Rubber female Mould}

Silicon rubber female mould is used for making the composite test specimens. For the preparation of silicon rubber mould we need some metallic specimen of the same dimension with some positive tolerance that we required for composite specimens.
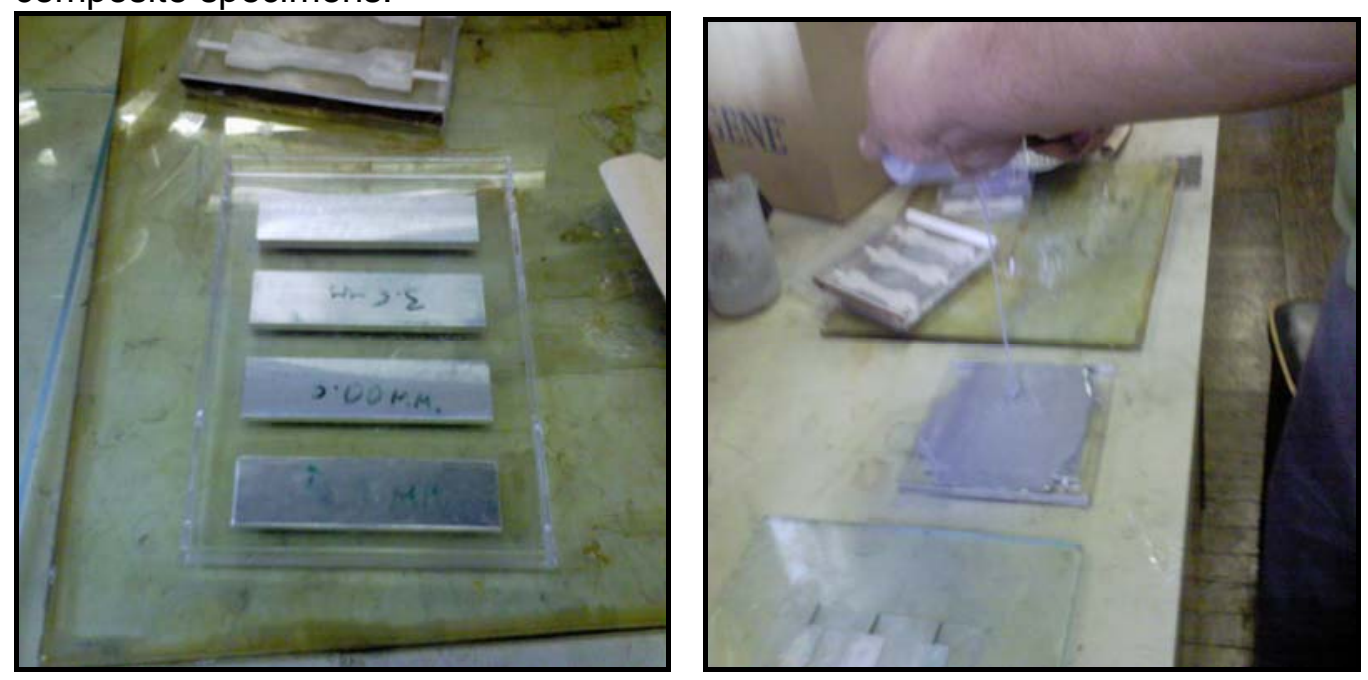

Fig .1. Silicon Rubber Female Mould preparation

For silicon rubber mould first mix 100\% High performance silicon rubber, Bentley Chemicals type RHODOSIL RTV (3450), quantity 103.77gm and hardener 10\% B50Black RTV (3450) black curing agent, quantity 10gm in one jar for 5-7 minutes. Place the metallic specimens in four sides cover tray and glued each metallic specimen at the bottom with tray. In the final stage pour the mixture in the tray in such a fashion that all the metallic specimens should 
completely dip in to the mixture. The procedure is shown in Fig.1. After 48 hours we get very fine silicon rubber female mould as shown in Fig.2. The resultant female mould will be used for making single short fibre composite test specimen.
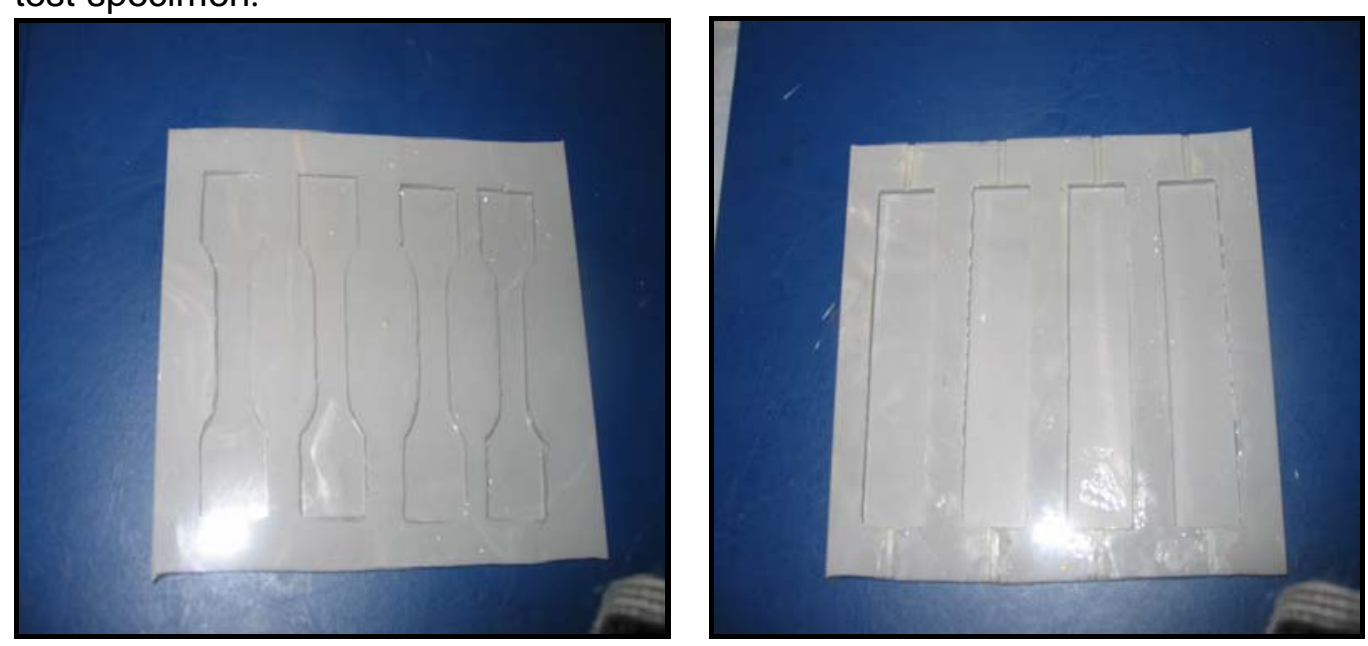

Fig .2. Final Shape of Silicon Rubber Female Mould

\subsection{Interphase Fabrication}

To study the effect of the interphase on composite performance a range of controlled interphase layers are applied to single glass fibre [17].

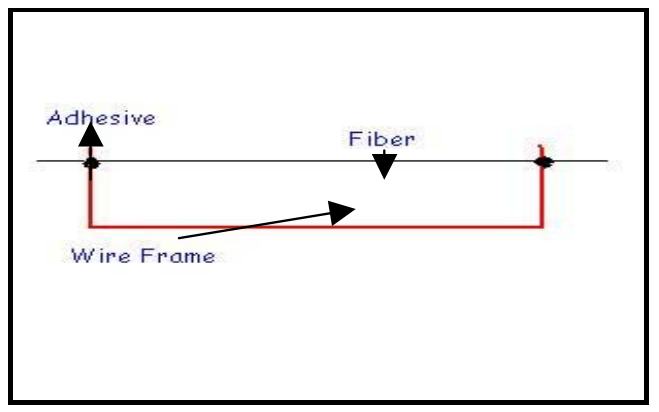

Fig.3. Mounting of individual fibre on wire frame [1]

Fibre is separated from the tow and mounted on the wire frames as described in Fig.3. Application of the interphase layer is carried out using a glass-stirring rod mounted on a clamped stand. Resin of the appropriate composition is prepared and degassing (temperature + vacuum) for 15 minutes of temp $50^{\circ} \mathrm{C}$ to reduce the viscosity and remove the air bubbles [1]. The Glass rod is then dipped into resin, clamped at the stand and the fibre passed through the resin on the rod. Using a sideways motion and introducing a slight shear achieve a uniform coating of the appropriate thickness achieved on the fibre surface. Cure the coated fibre at $80^{\circ} \mathrm{C}$ for one hour. Then measure the thickness of the coating using Scanning Electron Microscopy (SEM). In SEM measure the diameter of the fibre without coating embedded in the matrix at least four to five different places and takes the average value. Again measure the diameter of the coated fibre embedded in the matrix at least four to five 
different places and takes the average value. Substrate both values to find the thickness of the fibre. If coating is not sufficient to match the requirement then apply additional coatings of the same resin using the same procedure. Fig. 4 shows the coated fibre in a metallic stand after curing.


Fig.4. Coated fibre in a metallic stand after curing

\subsection{Short Fibre Specimen}

For the single short fibre composite specimen fabrication, it is very difficult to get the align fibre at the centre of the specimen. Best thing to draw a straight line inside the silicon rubber mould initially. Prepare the half mixture of $\underline{H Y} \underline{5052}$ with the same ratio, which mentioned before. Pour that mixture

\section{LY5052}

after degassing (No air bubbles) into the rubber female mould then cure the poured mixture within a mould for 10 minutes at $25^{\circ} \mathrm{C}$. Cure mixture in the mould become thick in the form of jelly. Put the single short fibre at the centre of semi-cured matrix as shown in Fig.5 (a). Now pour rest of the half of

$\frac{H Y 5052}{L Y 5052}$ on the semi cure mixture within the rubber mould as shown in Fig.5 (b), cured the whole mixture within the mould at $50^{\circ} \mathrm{C}$ for one hour and $80^{\circ} \mathrm{C}$ for four hours. After this keep the specimen in the same switch off oven for 8-10 hours or overnight for slow cooling.

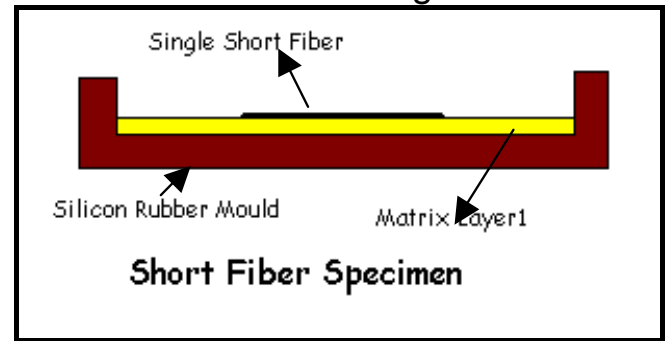

(a)

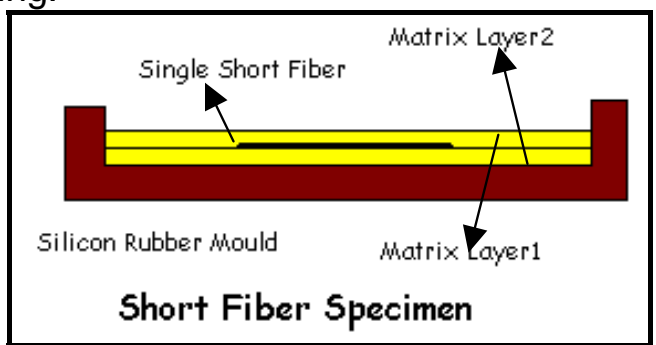

(b)

Fig.5. Embed technique of short fibre in specimen

\subsection{Specimen curing}

There are two methods of curing i.e. cold and hot curing. Cold Curing is preferable than hot curing but cold curing is time consuming method. Cold curing takes 7 days to cure the specimen at room temperature but due to this curing thermal residual stress should be skipped as shown in Fig.6 (a). Hot curing taking one hour at $50^{\circ} \mathrm{C}$ and four hours at $80^{\circ} \mathrm{C}$ and during this curing 
thermal residual stresses are developed within the specimen without loading as shown in Fig.6 (b) and that residual stresses play an important role in the failure of specimen. Thermal residual stresses can be minimized if cured the specimen at low temperature. Also the specimens which has large residual stress at the fibre end or fibre matrix interphase were discarded. See the effect of cold and hot curing on the specimen is shown in Fig.6.

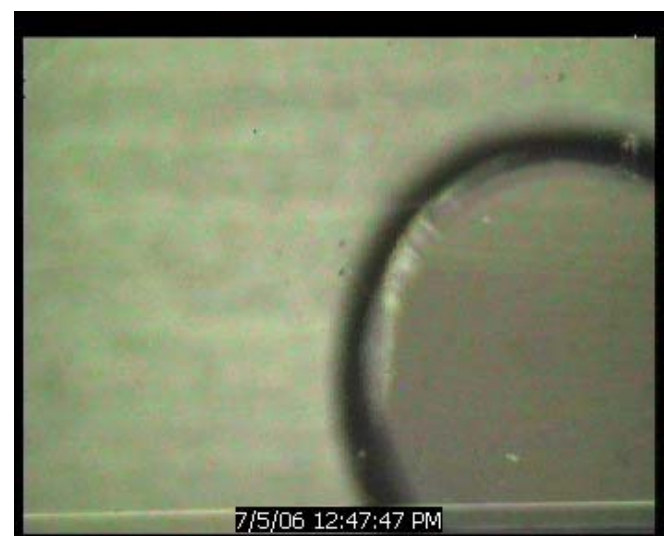

(a)

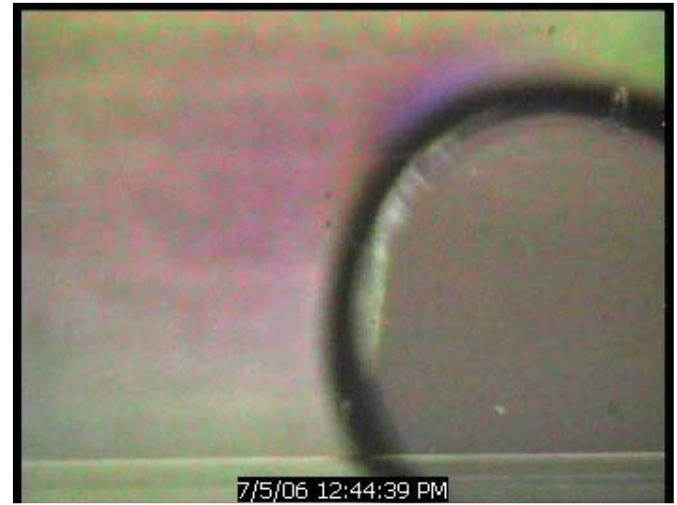

(b)

Fig.6. (a) No residual stresses due to Cold curing without loading (b) Residual stresses in the form of different colors contour due to hot curing without loading.

\section{Optical Microscopy}

Optical microscope is used to study the micro damages like debonding, fibre buckling, and fibre breakage under transmitted light because of transparent epoxy system.

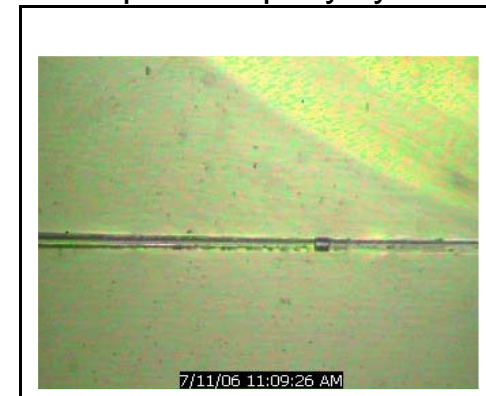

(a)

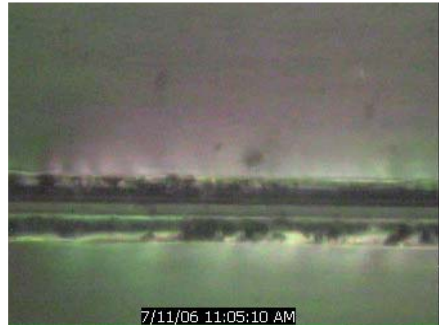

(d)

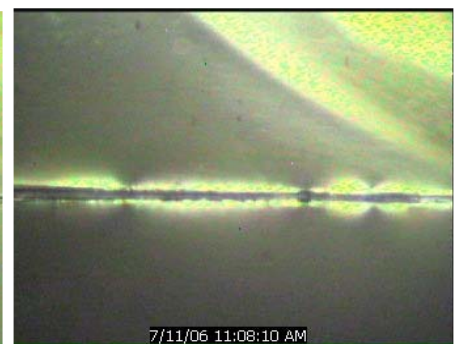

(b)



(e)

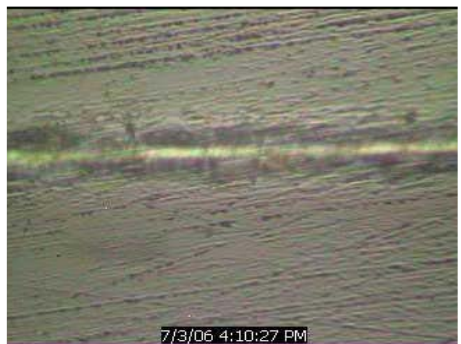

(c)



Fig.7. (a) Stress concentration on single short fibre test specimen with LM Interphase at small loading. Similarly Fig-7 (b) shows Stress concentration with HM interphase of same loading. (d), (e), (f) shows different phases of debonding along the fibre and especially at the centre under Compressive Loading. The birefringence pattern around the fibre is different because of LM and HM interphase. 
Photoelasticity use polarized light to capture the fibre breakage, fibre buckling and debonding at the same region. Fig.7 shows the micro damage phenomenon under compressive loading. Fig.7 (a) shows Stress concentration on single short fibre test specimen with LM Interphase at small loading. Similarly Fig.7 (b) shows Stress concentration with HM interphase of same loading. Fig.7(c), (d), (e), (f) shows different phases of debonding along the fibre and especially at the centre.

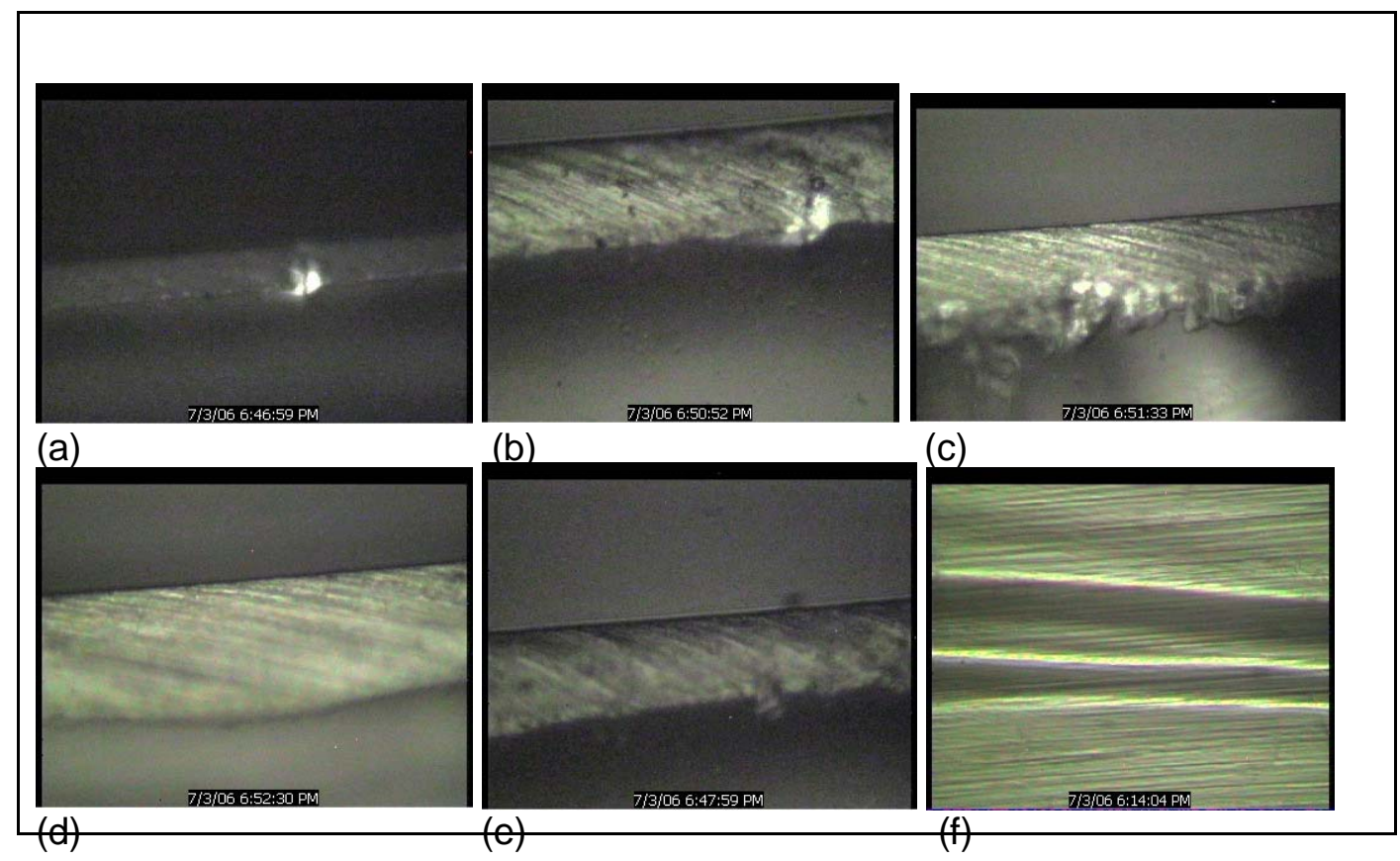

Fig.8. buckling and deformation phenomenon in fibre under compression, (a) bright region shows deformation start initially at a point, (b) zoom view of (a), (c) show due to gradual increase load, deformation penetrate longitudinally as well as radially, (d), (e), (f) shows different buckling view under different compressive loading.

\section{Fibre buckling/ twisting phenomenon in Photoelasticity}

Photoelasticity technique is used to see the stress effect in composite due to fibre buckling/twisting under different loading in different directions. For this single short Glass fibre embedded in matrix is axially loaded in compression. With the increasing compressive force on the short fibre will cause the buckling phenomenon. Along with determination of the critical load for the fibre, the maximum interfacial shear stress at any point in the epoxy matrix is determined using phase stepping photoelasticity. Fig.9 shows a fibrebuckling phenomenon under compression loading using optical microscopy. In photoelasticity Fig.10 shows isochromatic fringes near the same region of matrix where fibre buckled under different loading. i.e. $0.9 \mathrm{KN}$ and $1.5 \mathrm{KN}$. Isochromatic contour bar has 12 colours and each colour has some values of fringe orders. The stress concentration at the fibre/matrix interphase due to fibre buckling will move toward the matrix in radial direction away from the interphase due to gradually increase the load. So this will affect the efficiency of stress transfer from matrix to fibre. Fig.11 shows optical microscopy viewfibre twisting/buckling phenomenon simultaneously under compression. 
Fig.9. Optical Microscopic View of Fibre buckling under compression

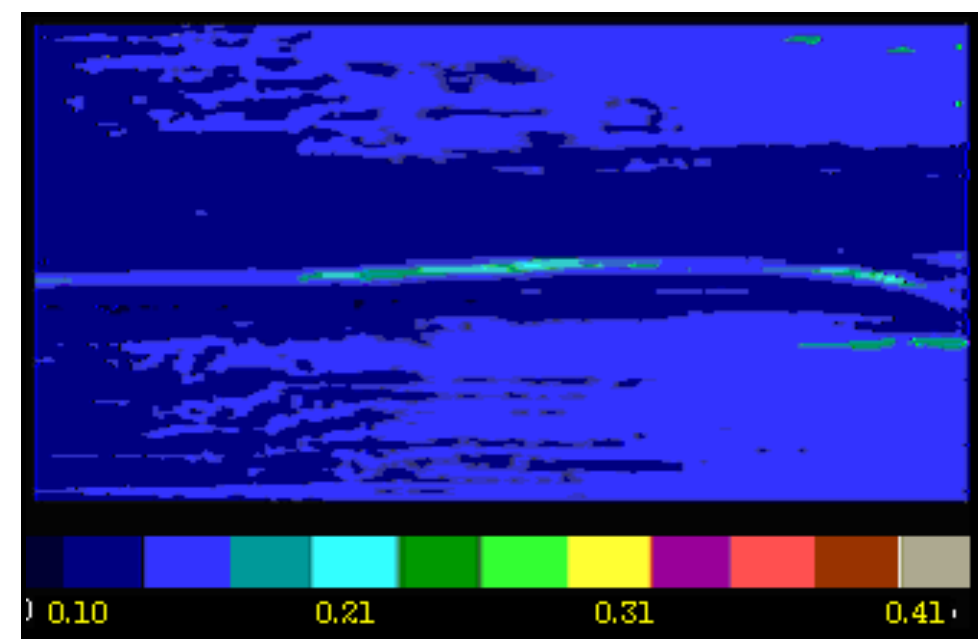

(a)

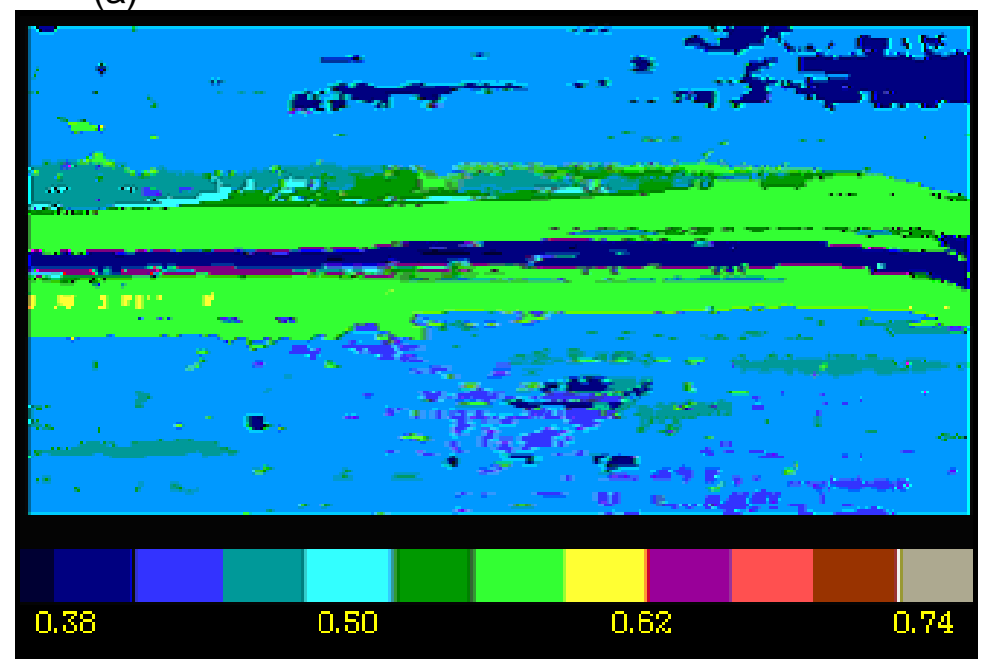

(b)

Fig.10. (a) Contour of isochromatic fringe order in the matrix due to fibre buckling at $0.9 \mathrm{KN}$ in compression. (b) Contours of fringe order in the matrix at $1.5 \mathrm{KN}$ 
Fig.11. Optical Microscopic View of Fibre twisting/buckling under compression

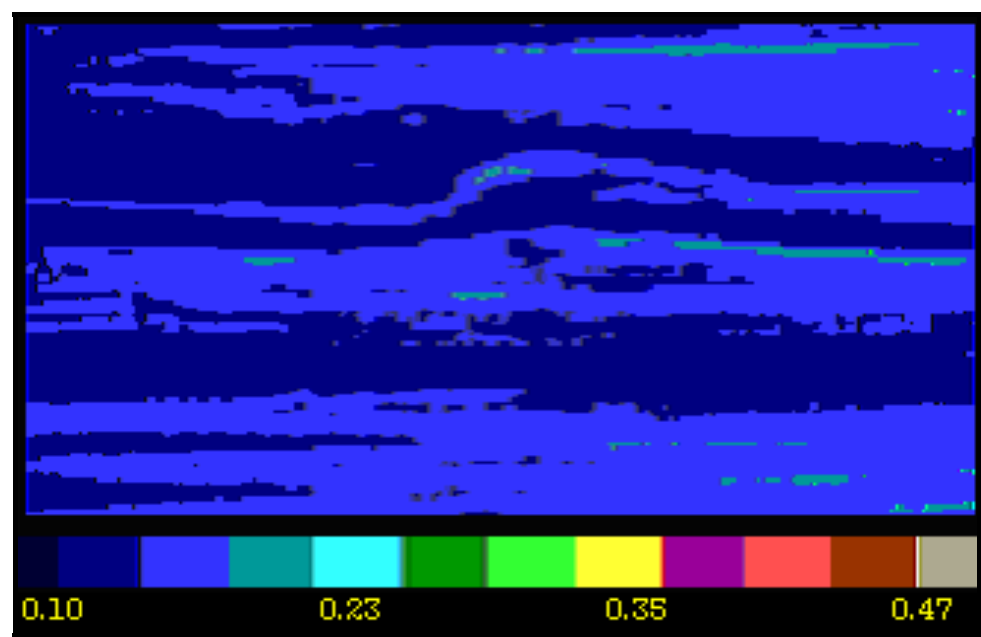

(a)

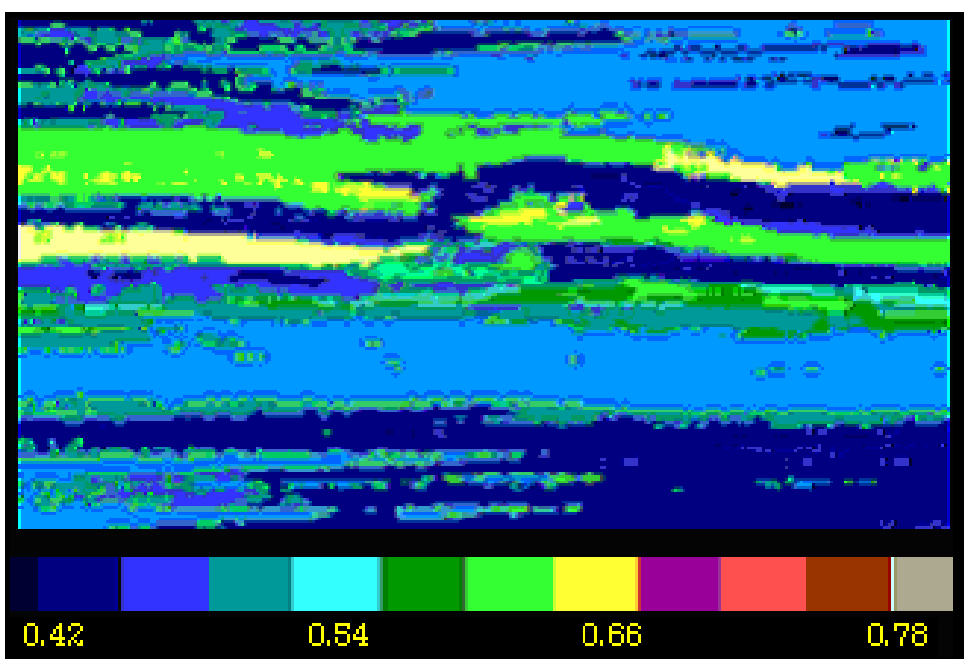

(b)

Fig.12. (a) Contour map of isochromatic fringe order in the matrix due to fibre twisting/ buckling at $0.9 \mathrm{KN}$ in compression. (b) Contours of fringe order in the matrix at $1.5 \mathrm{KN}$ 
Again this fibre twisting phenomenon effect the load carrying capability of fibre and at some increased load this fibre twisting will cause fibre failure. From Fig.12 the isochromatic fringes near the same area where the fibre buckled at $0.9 \mathrm{KN}$ and $1.5 \mathrm{KN}$. The important conclusion is that due to fibre buckling/twisting the interfacial debonding and yielding of the matrix may occurs near the Interphase [35]. So maximum value of interfacial shear stress occurs throughout the fibre in compressive loading, which may explain the appearance of continuous isochromatic fringe in the matrix near the twisting/buckling fibre as shown in Fig.12.

\section{Results and discussions}

\subsection{FEA Interphase (HM and LM) in compression}

\subsubsection{HM Interphase}

FEA model is meshed with different element types. Fibre and interphase is meshed with SOILD 45(8 node structural solid bricks element), matrix is meshed with SOLID92 (10 node tetrahedral element) [6]. To reduce the computational time, symmetrical model only 90 degree apart is used. The material used for HM Interphase has young's modulus 4GPa; matrix has 3.2GPa and glass fibre as reinforcement has 76GPa. Compressive Load is applied gradually in five steps of total load i.e. $1.5 \mathrm{KN}$ (Maximum).
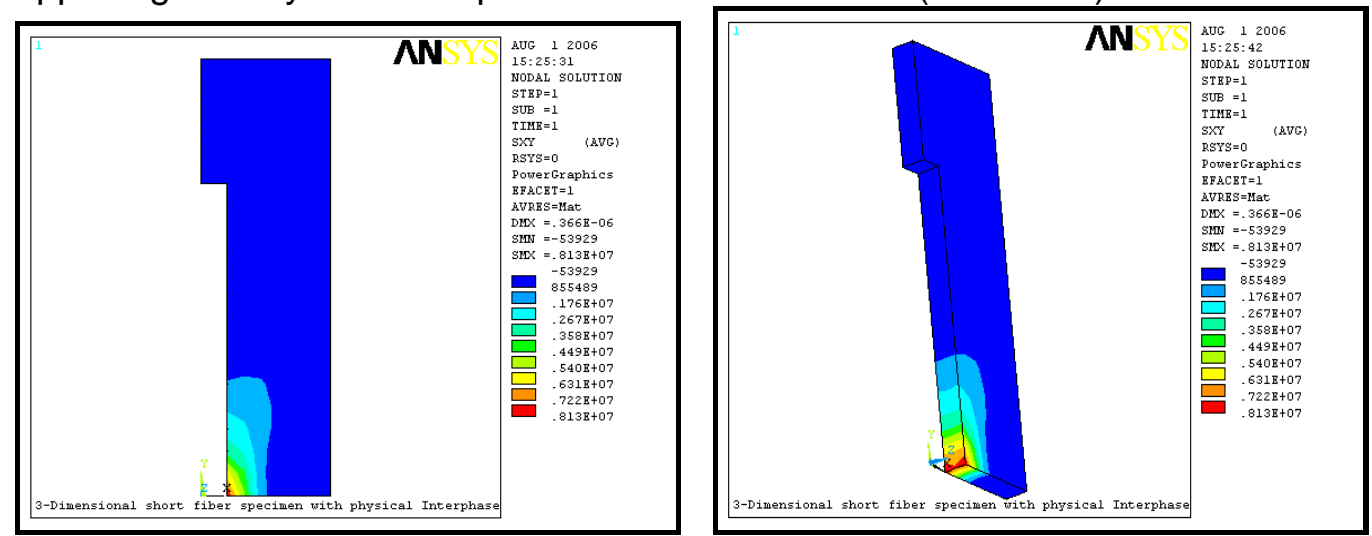

(a)



(b)

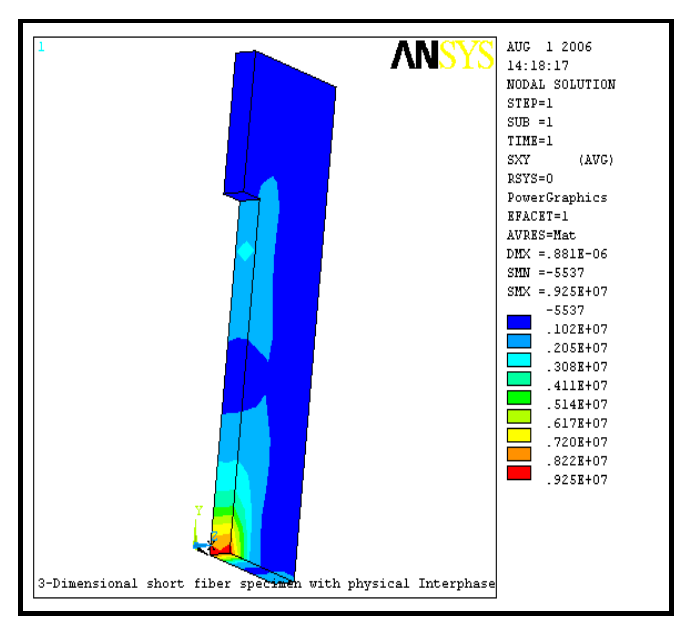



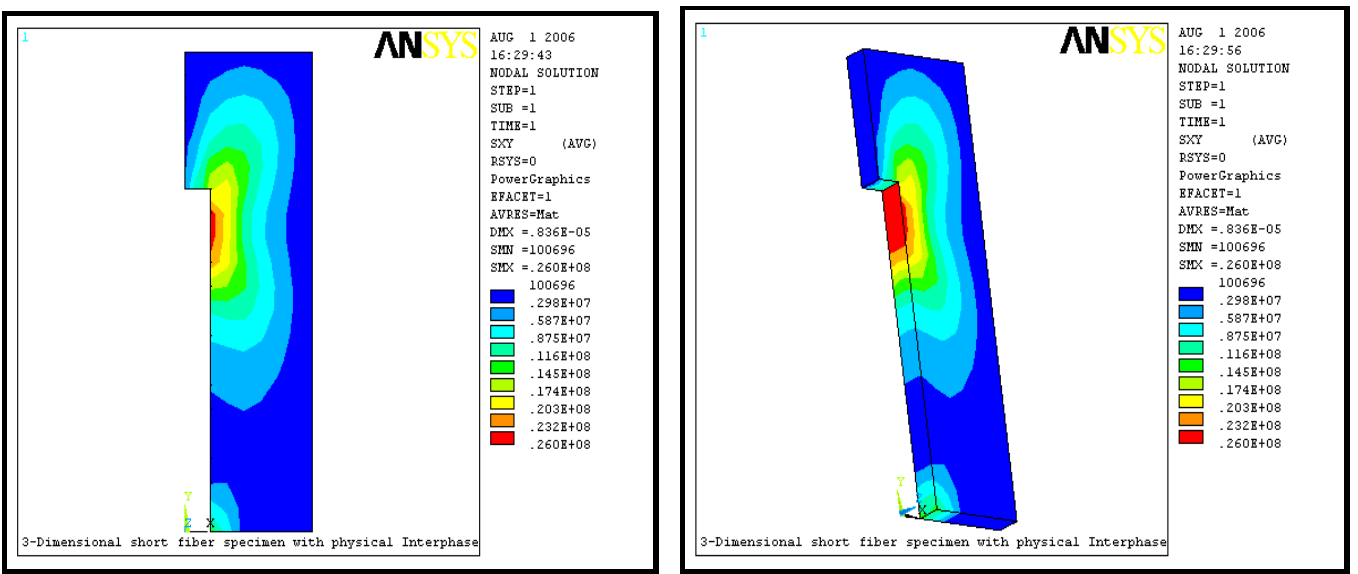

(c)

Fig.13. (a) FEA stress concentration in the matrix at the fibre centre due to gradually increasing load at $0.3 \mathrm{KN}, 0.6 \mathrm{KN}$ in compression (b) At $1.2 \mathrm{KN}$ small stress concentration appeared at the fibre end, (c) At $1.5 \mathrm{KN}$ the existing stress concentration value at the fibre end will further grow

Initially two steps i.e. $0.3 \mathrm{KN}$ and $0.6 \mathrm{KN}$ small stress concentration appeared at the fibre centre as shown in Fig.13 (a) and at $0.9 \mathrm{KN}$ this concentration intensity will increase at the fibre centre and penetrate into the matrix in radial direction. At the same time small concentration appeared at the fibre end as shown in Fig.13 (b). At load of $1.2 \mathrm{KN}$ and $1.5 \mathrm{KN}$ the stress concentrations at the fibre end will further increase.This fibre end stress concentration does not move towards the fibre centre due to HM interphase as shown in Fig.13(c). Within these step loading the interfacial shear stress value between fibre/matrix interphase and axial stress along the fibre is measured.

\subsubsection{LM Interphase}

Initially two steps i.e. $0.3 \mathrm{KN}$ and $0.6 \mathrm{KN}$ stress concentration appeared at the fibre centre but the interfacial shear stress value is lower than the HM interphase as shown in Fig.14 (a). At $0.9 \mathrm{KN}$ this concentration intensity increase at the fibre centre due to fibre buckling and at the same time small concentration appeared at the fibre end as shown in Fig.14 (b). At fourth step i.e. $1.2 \mathrm{KN}$ the stress intensity at the fibre end will grow and moves towards the fibre centre due to LM interphase as shown in Fig.14(c).



(a)

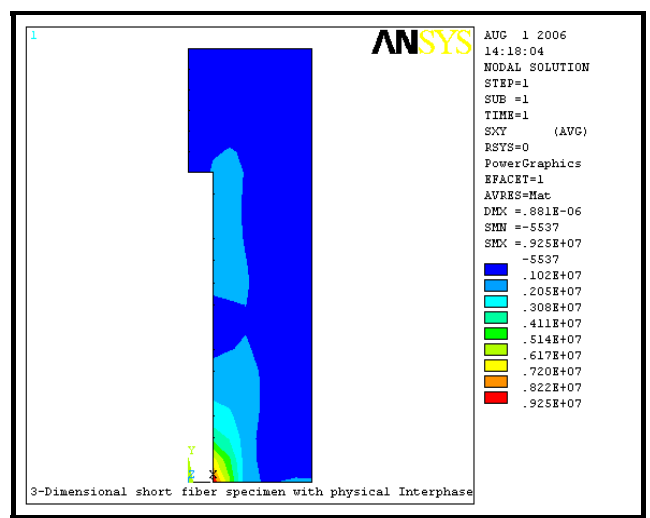

(b) 

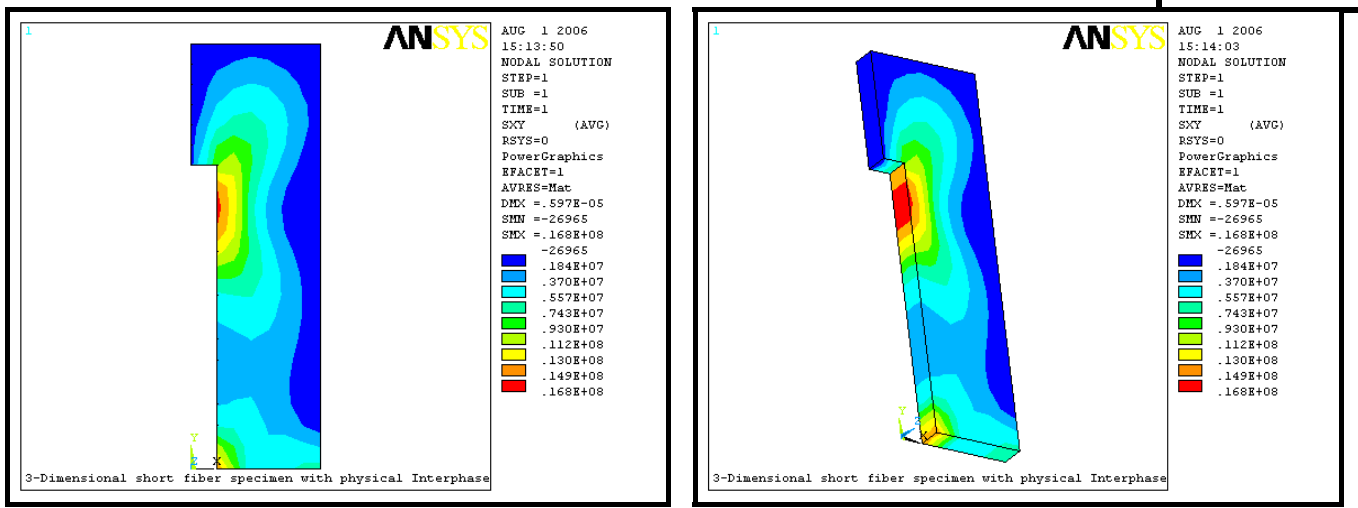

(c)

Fig.14. (a) FEA Interfacial shear stress value in the matrix at the fibre centre due to gradually increasing load at $0.3 \mathrm{KN}, 0.6 \mathrm{KN}$ in compression (b) At $0.9 \mathrm{KN}$ small concentration appeared at the fibre end and fibre centre concentration will grow, (c) At $1.2 \mathrm{KN}$ and $1.5 \mathrm{KN}$ the existing stress concentration value at the fibre end will further grow and move towards the centre.

At $1.5 \mathrm{KN}$ the fibre end stress concentration will further grow. But the maximum ISS value at $1.5 \mathrm{KN}$ is lower than the HM interphase.

\subsection{FEA Comparison of Interfacial Shear stress between Interphase (LM and $H M$ )}

FEA Comparison of the corresponding ISS between the LM and HM Interphase at compressive load $0.3 \mathrm{KN}, 0.6 \mathrm{KN}, 0.9 \mathrm{KN}$ and $1.5 \mathrm{KN}$ is shown in Fig.15. There is slightly variation in shear stress value due to different nature of interphase. In first two load steps in HM and LM interphase i.e. at 0.3KN and $0.6 \mathrm{KN}$ stress concentration appeared at the fibre centre. At load $0.9 \mathrm{KN}$ this concentration will further grow in same location.

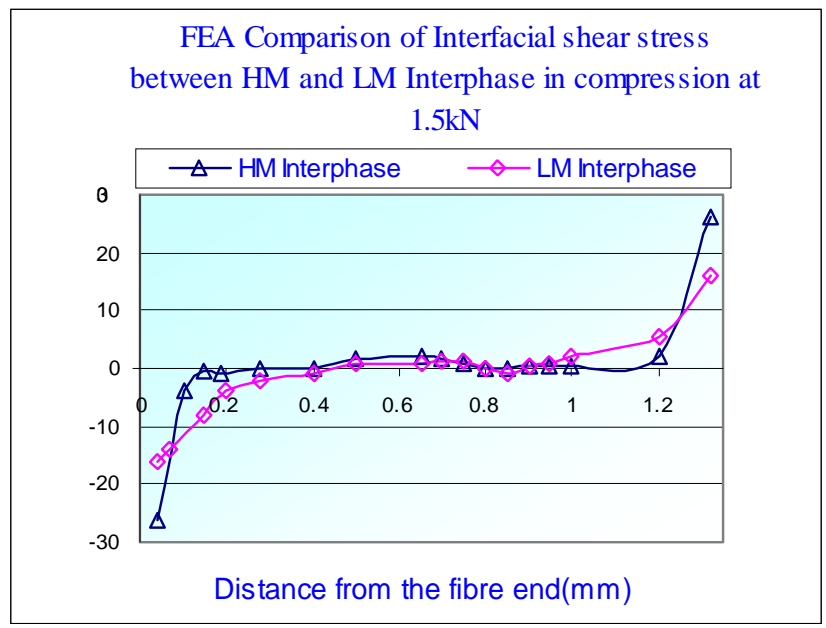

Fig.15. FEA Comparison of Interfacial Shear Stress profiles between (LM and HM) for an applied compressive load 1.5KN 


\subsection{Photoelasticity Interphase (LM and HM) in Compression}

\subsubsection{HM Interphase}

Epoxy828 is used as a resin and BDMA/NMA is used as a hardener in $\mathrm{HM}$ Interphase. For making $\mathrm{HM}$ interphase around the fibre all these ingredients are mixed in the weight ratio 100gm (Epoxy828), 90gm (NMA) and $1 \mathrm{gm}$ (BDMA). Interphase coating procedure is same as mentioned previously. Load will be applied in five steps. i.e. loads starts at $0.3 \mathrm{KN}, 0.6 \mathrm{KN}, 0.9 \mathrm{KN}$, $1.2 \mathrm{KN}$ and the highest load will be $1.5 \mathrm{KN}$. To observe the effect of $\mathrm{HM}$ interphase around the fibre on the stress transferring efficiency under gradually increasing load, the behaviour of composite in compressive loading is bit different in tensile loading. In compressive load the debonding start at the fibre centre due to fibre buckling with in initial load and stress region appeared in this buckled area. With the gradually increasing load this centre stress concentration penetrate in matrix in radial direction and small stress concentration around the fibre end. Fig.16 (a) is the phase of debonding and Stress concentration in the fibre centre due to gradually increase load i.e. $0.3 \mathrm{KN}, 0.6 \mathrm{KN}, 0.9 \mathrm{KN}, 1.5 \mathrm{KN}$ and Fig.16 (b) shows the zoom view of fibre centre at load 1.5KN. Fig.17 (c), (d) shows the left and right side of fibre ends at load $1.5 \mathrm{KN}$, but this stress concentration is not too much higher than in tensile loading. So the maximum interfacial shear stress occurs at the fibre end but before this the stress concentration appeared at the fibre centre due to fibre/matrix debonding in compression.

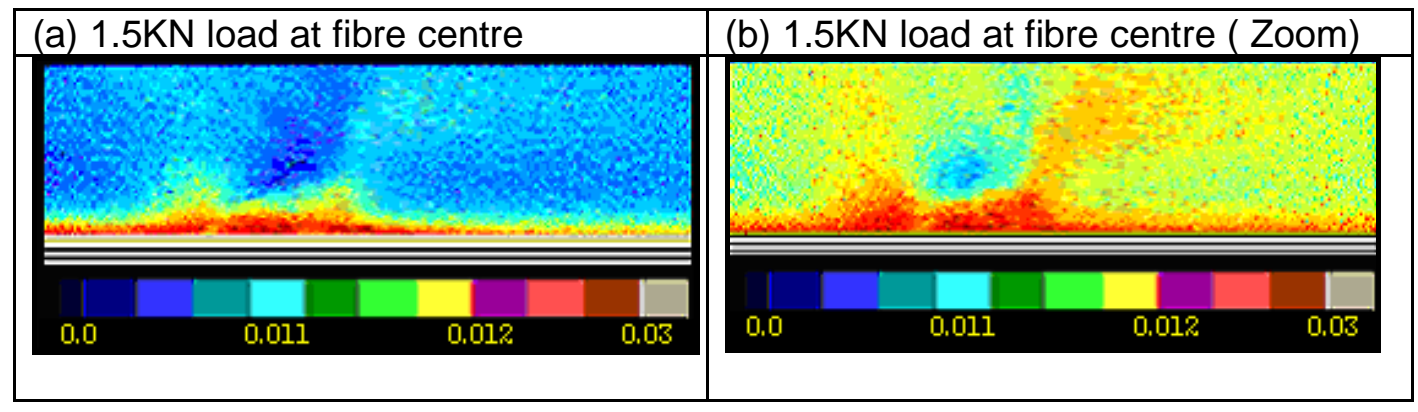

Fig.16. (a) Contour map of isochromatic fringe order in the matrix at the fibre centre due to gradually increasing load at $0.3 \mathrm{KN}, 0.6 \mathrm{KN}, 0.9 \mathrm{KN}$ and $1.5 \mathrm{KN}$ in compression, (b) contours (Zoom view) of fringe order in the matrix at fibre left end at load $1.5 \mathrm{KN}$ in compression.



Fig.17. (c), (d) Contours map of isochromatic fringe order in the matrix at fibre left and right end at load $1.5 \mathrm{KN}$ in compression 
So the important conclusion is that in compression the stress concentration appeared at the centre of fibre initially and the fibre end stress concentration isn't moves towards the fibre centre due to HM interphase. In compressive loading there is no indication of fibre failure even if the fibre has buckled/twist repeatedly at the centre. In photoelasticity these isochromatic fringe patterns are used to calculate the interfacial shear stress at the fibre/matrix interphase.

\subsubsection{LM Interphase}

In compressive testing of single short fibre composite, Unsized Glass fibre has an average diameter of $80 \mu \mathrm{m}$ is used as single short fibre. For coating Epoxy (Shell Epicote 828) is as a resin and Nadic Methylene Anhydride (NMA) as a hardener, Araldite GY 298 as a Flexibilizer is used as LM Interphase on this glass fibre. For making LM interphase around the fibre all these ingredients are mixed in the weight ratio 70gm (Epoxy828), 50gm (Araldite GY 298) and curing agent 55gm Nadic Methylene Anhydride (NMA). Interphase coating procedure is same as mentioned previously. Coated fibre is cured for four hours at $80^{\circ} \mathrm{C}$ and three hours at $130^{\circ} \mathrm{C}$ for post curing. For slow cooling it will essential to place the cured fibre within same switch-off oven over night. Load will be applied in five steps. i.e. loads starts at $0.3 \mathrm{KN}, 0.6 \mathrm{KN}, 0.9 \mathrm{KN}$, $1.2 \mathrm{KN}$ and the highest load will be $1.5 \mathrm{KN}$. At initial load the stress concentration zone appeared at the fibre centre due to compressive load .Due to gradual increasing load the intensity of this fibre centre stress concentration increases and will penetrate in matrix in radial direction as shown in Fig.18 (a) and Fig.18 (b) shows the zoom view of fibre centre at load 1.5KN. At the same time some stress concentration appeared at the fibre ends but both stress value at the fibre centre and fibre end will be lower than the HM interphase.

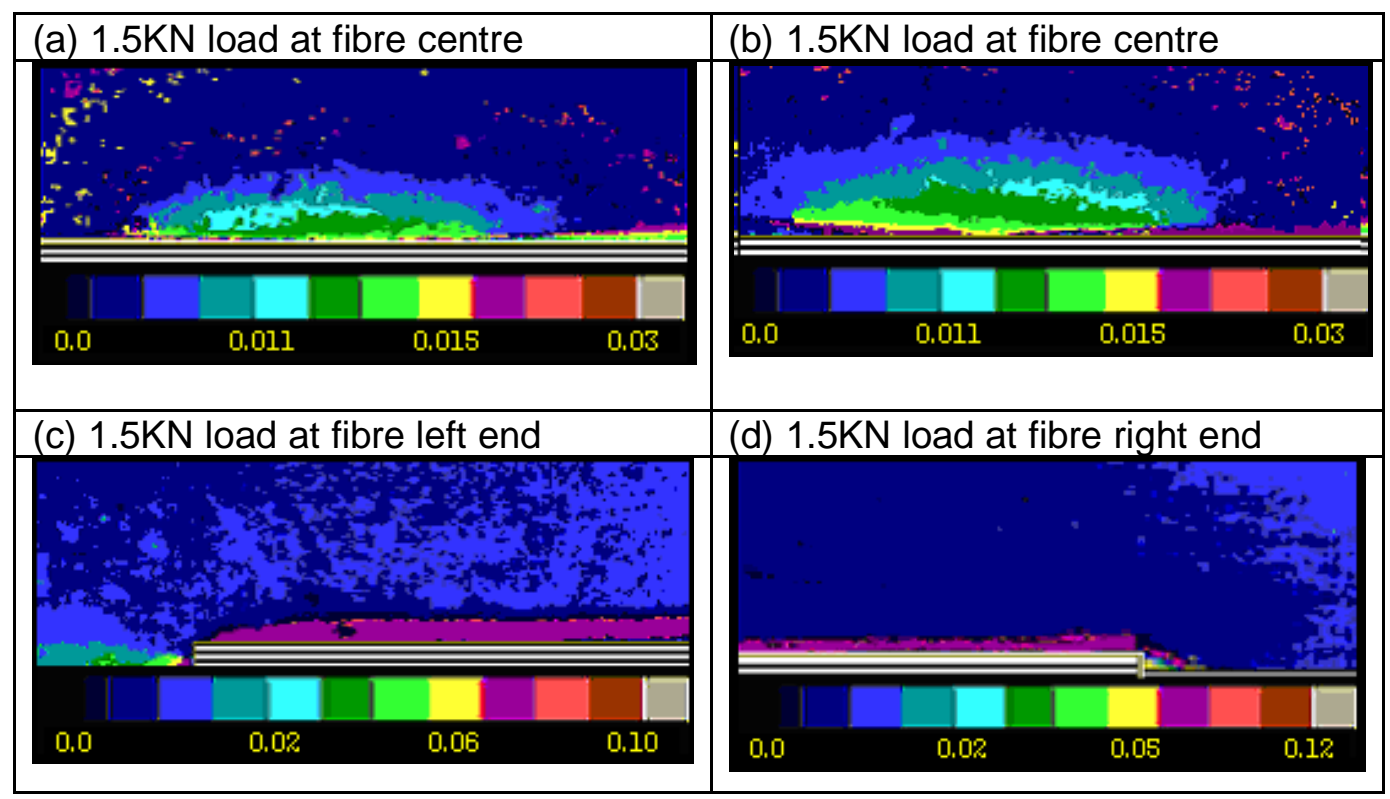

Fig.18. (a) Contour map of isochromatic fringe order in the matrix at the fibre centre due to gradually increasing load at $0.3 \mathrm{KN}, 0.6 \mathrm{KN}, 0.9 \mathrm{KN}$ and $1.5 \mathrm{KN}$ in compression, (b) contours (Zoom view) of fringe order in the matrix at fibre centre at load $1.5 \mathrm{KN}$ in compression and (c),(d) Contours of fringe order in the matrix at fibre left and right end at load 1.5KN in compression 
Due to further increasing load the intensity of fibre end stress concentration increases and will moves towards the fibre centre due to LM interphase as shown in Fig.18(c), (d). Again the fibre end stress concentration intensity is not too much as compared to HM interphase. From these photoelasticity plots it can be seen that in LM interphase there is less stress concentration at the fibre end as compared to HM interphase.

\subsection{Comparison of Interfacial Shear Stress between Interphase (LM and $\mathrm{HM}$ ) in compression}

Comparison of the ISS between the LM and $\mathrm{HM}$ Interphase in compression at $1.5 \mathrm{KN}$ is shown in Fig.19. In compressive loading LM interphase has low ISS value as compared to HM Interphase. It can be seen that in both LM and HM stress concentration occurs at the fibre centre first and than at the fibre end after some load.

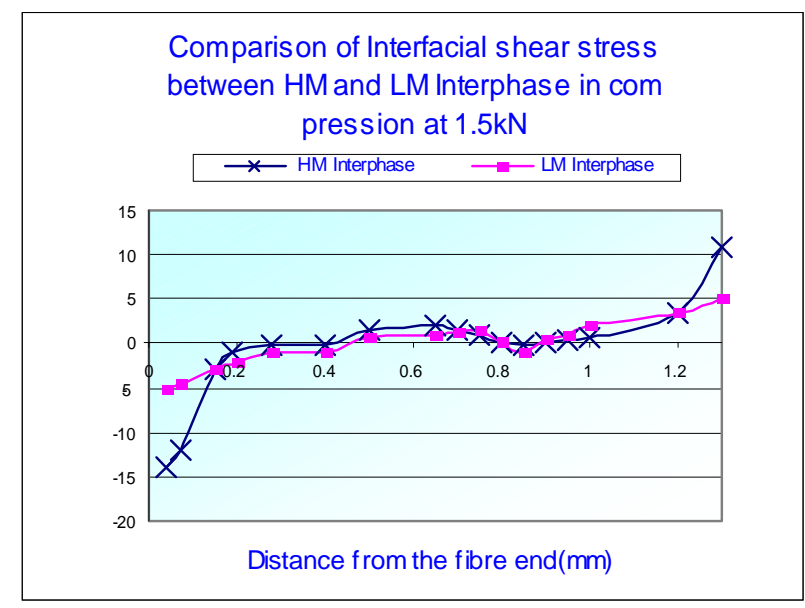

Fig .19. Graphical Comparison of Interfacial Shear Stress profiles Between (LM and HM) for an applied compressive load 1.5KN

But in LM this stress concentration intensity moves towards the centre due to load and this stress concentration moving phenomenon is not appeared in HM interphase. There is slightly variation in shear stress value due to different nature of interphase. No interfacial failure is observed because the maximum load lower than that required for fibre failure.

\subsection{FEA and Photoelasticity of HM interphase in Compression}

Comparison of interfacial shear stress between the FEA and photoelasticity of HM interphase in compressive loading is shown below in Fig.20. Interfacial shear stress vs. fibre length profile is obtained at load $0.3 \mathrm{KN}, 0.6 \mathrm{KN}$ and $0.9 \mathrm{KN}$. Major trend is same in both FEA and Photoelasticity curve i.e. stress concentration will be appeared initially at the fibre centre because of fibre buckling in compressive loading. Due to gradually increasing load fibre centre stress concentration will increase further and at the same time stress concentration appeared at the fibre end. This fibre centre stress 
concentration will penetrate into the matrix in radial direction as shown in ANSYS plot of Fig.13 and Photoelasticity plot of Fig.17.



Fig.20. Comparison of Interfacial Shear Stress profiles between FEA and Photoelasticity of HM interphase

\subsection{FEA and Photoelasticity of LM interphase in Compression}

Comparison of interfacial shear stress between the FEA and photoelasticity of LM interphase in compressive loading is shown below in Fig.21. Interfacial shear stress vs. fibre length profile shown below is obtained at load $0.3 \mathrm{KN}, 0.6 \mathrm{KN}$ and $0.9 \mathrm{KN}$. Major trend is same in both FEA and Photoelasticity curve i.e. stress concentration is appeared initially at the fibre centre because of fibre buckling in compressive loading. After some load fibre centre stress concentration will increase further and at the same time small stress concentration appeared at the fibre end. But with the gradually increasing load this fibre centre stress concentration will penetrate into the matrix in radial direction.



Fig .21. Comparison of Interfacial Shear Stress profiles Between FEA and Photoelasticity of LM Interphase 
At the same time stress concentration at the fibre end is gradually increasing and become higher than the fibre centre. There is significant variation in interfacial shear stress value between FEA results and photoelasticity results. One important conclusion in LM interphase is that the fibre end stress concentration will move towards the fibre centre due to gradually increasing load as shown in ANSYS plot of Fig.14 and Photoelasticity plot of Fig.18.

\section{Conclusions}

The objective of the current work is to see compressive behaviour of (GFRP) Glass Fibre Reinforced Polymers composite at Fibre/Matrix Interface/interphase. This research work consists of finite element model for a single short fibre with two interphase (LM and HM) modeled in the matrix and compares their interfacial shear stress in compression. Single short fibre test specimen is fabricated with LM and HM interphase embedded in a polymeric matrix, experimental testing performed. In this experiment Phase stepping photoelasticity technique is used to calculate the interfacial shear stress in tension and compression. Finally the results from FEA interfacial shear stress (LM and HM) compared with photoelasticity interfacial shear stress value (LM and $\mathrm{HM}$ ) in compression.

It is observed during compressive loading in both FEA and photoelasticity that the stress concentration location and stress moving phenomenon is same. It can be seen that in both FEA and Photoelasticity the stress concentration appeared initially at the fibre centre in compression. Due to gradually increasing load the intensity of the fibre centre stress concentration increases and penetrate in the matrix in radial direction. At the same time stress concentration appeared close to the fibre end. Due to further increasing load the fibre end concentration moves towards the centre in LM interphase but this stress moving phenomenon is not appeared in HM interphase.

There is some variation in net interfacial shear stress value of both (LM and $\mathrm{HM}$ ) interphase in both FEA and photoelasticity due to different nature (hardlsoft) interphase in compression. The final comparison is in between the results of FEA and Photoelasticity. Interfacial shear stress values obtained by FEA of (LM and HM) interphase are compared with the interfacial shear stress values obtained by photoelasticity of (LM and HM) interphase in compression. It was observed that the interfacial shear stress value of both FEA and photoelasticity is higher in HM interphase as compared with the LM interphase. The research work can be considered as millstone in the study of compressive behavior of fibre/matrix interphase in single short fibre composite providing basic concepts, experimental constraints and better compatibility between FEA and photoelasticity results. Most important conclusion in compressive loading is that due to fibre misalignment and buckling, the efficiency of stress transfer is affected before the interfacial failure at the fibre end at minimum load. Therefore Fibre/Matrix interphase changes the composite compressive strength. 


\section{References}

[1] Lane (April 2000), 'Studies of load transfer through an interface /interphase in fibre reinforced composites. Dep't of Engineering Materials, University Of Sheffield.

[2] Fiedler, B. and Schulte, K., Photo-elastic analysis of fibre-reinforced model composite materials, Composites Science and Technology, 57(1997), 859-867.

[3] Netravali, A.N.; Henstenburg, R.B.; Phoenix, S.L.; Schwartz, P., Interfacial Shear strength studies using single fibre filament composite test Part1: Experiments on graphite fibre in epoxy, Polymer composites, 10(1989),226-241.

[4] Anbu Clemension Johnson (Dec 2003), Modeling Of stress transfer through an interface/interphase in Fibre reinforced polymer composite, Dep't of Engineering Materials, University Of Sheffield.

[5] Hull, D. and Clyne, T.W., An Introduction to Composite materials, Second Edition, Cambridge University Press, Cambridge, 1996.

[6] ANSYS Product Literature. ANSYS Europe, Wyvols Court, Swallowfield, Reading, UK.www.ansys.com. 